\title{
Electricity detection system based on power line carrier communication
}

\author{
Xin-Yu Yang ${ }^{\dagger}$, Lin Zhang and Qiang Zhang, \\ School of Air and Missile Defense, \\ Air Force Engineering University, Xi'an, China \\ ${ }^{\dagger}$ E-mail: Yangxinyu_2004@163.com \\ Xin-Yu Yang and Qi-Shuang Ma \\ School of Automation Science and Electrical Engineering, \\ Beijing University of Aeronautics and Astronautics, Beijing, China
}

\begin{abstract}
In order to reduce communication invest, the electricity detection system based on power line communication (PLC) was studied in this paper. At first, the research status of PLC technology and application prospects was expounded. Then, the overall design of the system and modules was introduced. Next, the working principle of each module respectively was analyzed, mainly in the power line communication module, power detection module and the computer software based on MODBUS. Finally, the electric energy testing system was built, and achieves accurate detection data of electrical equipment by monitor computer.
\end{abstract}

Keywords: Power line communication; Power detection; monitor system.

\section{State of Research}

Power line communication (PLC) is a kind of the communications technology which utilizes the power line as transmission medium. Since putting forward, it has been widely used in various aspects, such as remote signal relay protection, remote monitoring of the power supply device in power systems, remote intelligent meter reading system, the remote monitoring of intelligent home appliances, access to the Internet, etc. As a kind of advanced technology, PLC technology has a broad application prospect and potential market. In the domestic, the State Grid Corporation of China has included this technology on the important research subject, which reflects the demand for this technology and related communications equipments. It will promote the research and application of the technology in maximum. Many foreign research institutions and company earlier carried out the technology research and test too. As attached so much attention to this technology in many organization, it is enough to reflect the importance of the technology and application prospects. 
But it also has some disadvantages in this technology. It mainly shows in that all sorts of electric equipment of access and disconnect is random, so the output impedance of sender device and the input impedance of receiver are difficult to match with the impedance of the circuit in data transmission, which leads to serious attenuation of communication signals in the transmission. Since noise interference of electrical equipment, it needed for further in-depth study to achieve high speed and reliable data transmission. So, the electricity detection system based on PLC was studied in this paper as an entirely system.

\section{Overall Design}

The block diagram of the whole electricity detection system based on PLC was seen as figure 1 . The system mainly consists by host computer and slave computer two levels system. A new type of multifunctional digital wattmeter with single chip UPD78F9224 and appropriative measuring chip RN8209 was designed in this paper, which regards as slave computer. In monitor terminal, the host computer receives the data and shows by special software system. Between two levels, it finishes data transmission by using PLC module HPLCS520F. Because of the same interfaces, it brings out easily extensibility.

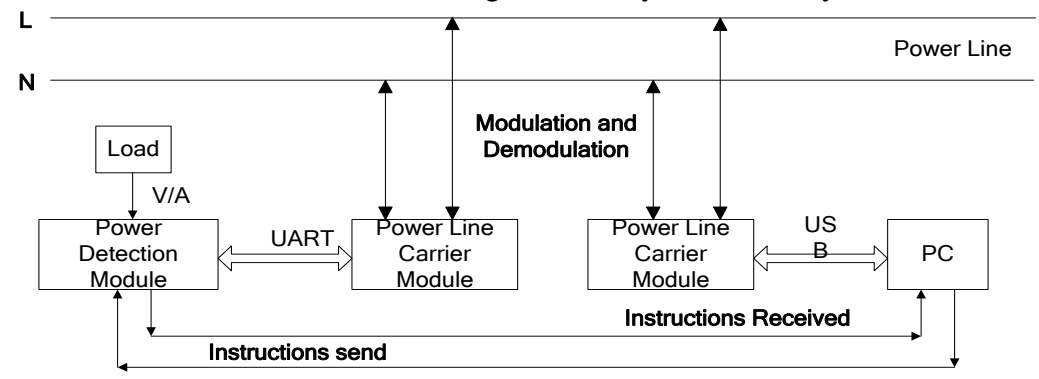

Fig.1 System function framework

\section{The Electricity Parameter Detection Module}

\subsection{Measuring and communication principle of electricity parameter}

Measuring circuit with the chip RN8209 was seen as figure 2. According the circuit diagram, there are three ADC channels, which are used to measuring three-phase current, zero line current and three-phase voltage. The circuit also has the process circuits of protecting and damping. In addition, the load circuit in charge of changing single is balanced in all range, and finish measuring accurately. Visiting measure chip by SPI, the MCU (Micro Control Unit) will read active power, reactive power and others, and storage homologous data into CSI24WC256. After that, it will show these data at real-time after calculating. 
Fig.2 Power detection circuit

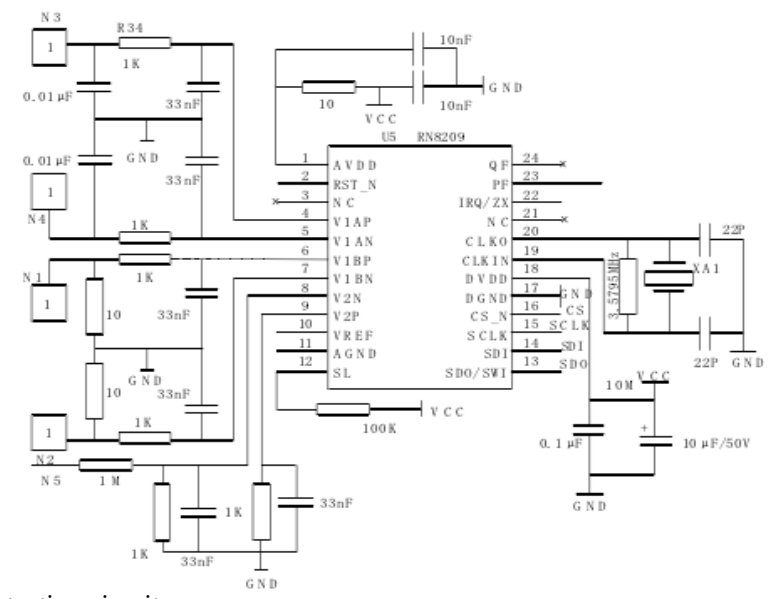

\subsection{The module communication protocol}

It adopts MODBUS protocol in electricity parameter detection module. MODBUS protocol can provide these service by function code, which has made it available to correspond among millions of automated electric equipments. Since the Internet keeps its system port 502 of TCP/IP be able to visit MODBUS, so MODBUS protocol uses widely in measurement. This protocol has two serial transmitting model (RTU and ASCII), which have defined the transmission of packet domain on the line, and decided how the information is translated into message and decoded. To accomplish the interoperability between equipments, it is necessary to use the RTU, in which include two hexadecimal character each byte. The model is able to get higher data density, and its default check model must be even-parity. The CRC verification will check the content of the all message, no matter there is or not even-odd check in the message.

\section{The Power Line Carrier Communication Module and its Principle}

Shown as figure 3, classical PLC circuit was adopted in the system. The carrier communication circuit is composed by MCU, interface circuit, modulation circuit and power line coupling interface circuit. According to the principle of frequency shift and division, it modulates the original signal once or more in accordance with different transmission bandwidth and facility. The frequency of communication is greater than $100 \mathrm{kHz}$ in general, so it can avoid the interference of $50 \mathrm{~Hz}$ power current. After modulating and amplifying by the carrier communication circuit, the digital signal will be loaded on the power line from signal coupling network. In the other side of the power line, the carrier communication circuit demodulates the signal in the same way. 


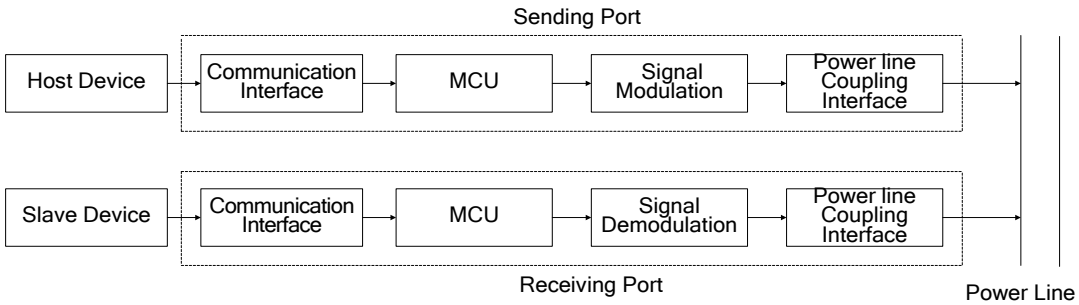

Fig.3 The basic principles of power line carrier communication

Frequency-shift keying (FSK) modulation is the most widely used in modulating mode, which delivers the digital message by changing frequency of the carrier wave. For incoherent FSK modulation, it's fully discrete transfer between instantaneous frequency, called spatial frequency and mark respectively. In the coherent FSK modulation, the output phase modulation signal with continuous change. Binary baseband signal was transformed to FSK signal by keying frequency modulation in computer for data transmission. On the receiving port, FSK signal will be demodulating into the corresponding identification binary digital signal by computer. Although the FSK technology appears earlier, it is still reliable and efficient in modulation and demodulation.

In this paper, the chip HLPLCS520F used as the key MCU of modulation and demodulation circuit in the PLC. It integrates with high speed digital signal processor (DSP) and FSK modem. The modem has high sensitivity and strong anti-interference, which can adapt to the complex channel environment of power line. The principle of the modem is shown in figure 4. It is mainly composed of sending circuit, receiving circuit, coupling circuit, filter circuit, crystals circuit, power circuit programming interface circuit.

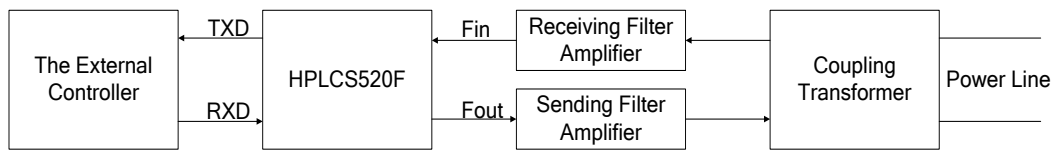

Fig.4 The PLC module block diagram

\section{Power Detecting Software System based on MODBUS}

MFC was been used as the host PC software development platform in the system, the main function includes: controlling contactor status, scanning and displaying the running state of the contactor. Instruction is requested by the host machine, and the slave machine received and responded accordingly. The slave machine did not send data initiatively, and could not communicate between each other. The flow chart of the software is shown as figure 5 .

Software main function is to monitor the status of the load, and reflect its electrical parameters in real time. The host sends instructions to the slave 
machine, the slave makes corresponding responses after receiving the instruction, reads the data in the memory and sends it back to the host. The register address and data processing method of electrical parameters shown as table 1 .

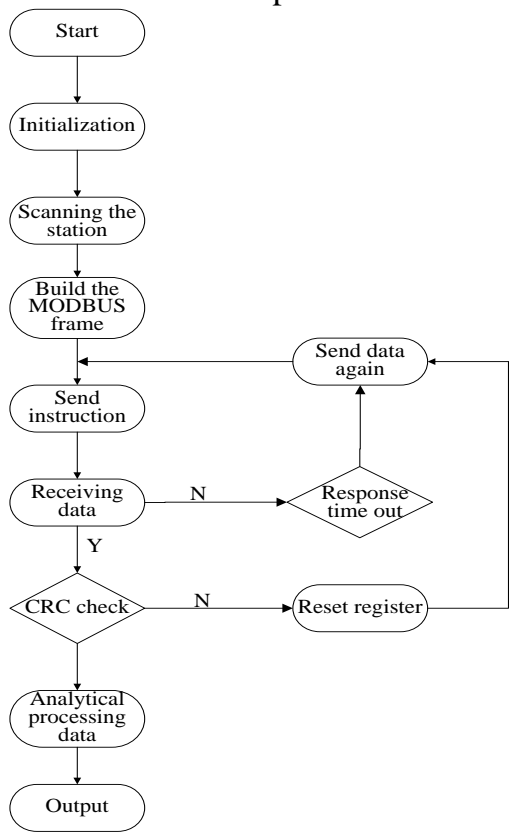

Fig.5 Flow chart of PC communication software

Tab. 1 the register address of MCU and data processing method of electrical parameters

\begin{tabular}{|c|c|c|c|c|}
\hline $\begin{array}{c}\text { Serial } \\
\text { number }\end{array}$ & Name & $\begin{array}{c}\text { Register } \\
\text { address }\end{array}$ & $\mathrm{R} \& \mathrm{~W}$ & Instructions \\
\hline 1 & Voltage & $0048 \mathrm{H}$ & $\mathrm{R}$ & $\begin{array}{c}\text { Unsigned number, value=DATA } / 100 \text {, } \\
\text { unit: } \mathrm{V}\end{array}$ \\
\hline 2 & Current & $0049 \mathrm{H}$ & $\mathrm{R}$ & $\begin{array}{c}\text { Unsigned number, value =DATA/100, } \\
\text { unit: A }\end{array}$ \\
\hline 3 & Active power & $004 \mathrm{AH}$ & $\mathrm{R}$ & $\begin{array}{c}\text { Unsigned number, value }=\text { DATA, unit: } \\
\text { W }\end{array}$ \\
\hline \multirow{2}{*}{4} & \multirow{2}{*}{$\begin{array}{l}\text { Active } \\
\text { electrical } \\
\text { energy }\end{array}$} & 004BH & $\mathrm{R}$ & \multirow{2}{*}{$\begin{array}{c}\text { Unsigned number, value }=\mathrm{DATA} / 3200, \\
\text { unit: } \mathrm{kWh}\end{array}$} \\
\hline & & $004 \mathrm{CH}$ & $\mathrm{R}$ & \\
\hline 5 & Power factor & 004DH & $\mathrm{R}$ & Unsigned number, value $=$ DATA $/ 1000$ \\
\hline
\end{tabular}

Through special MODBUS debug software, it will debug the read and write module. In this paper, the electric energy metering baud rate is set to $4800 \mathrm{bps}$, the check digit is NONE, the data bit is 8, the stop bit is 1 , and the device address is 01. According to the MODBUS communication format and the distribution of module register address, it will read and write the module register. 
It can test the function of sending and receiving by serial debugging assistant software. On the hardware, the two USB port of PC connect each other directly by $\mathrm{CH} 340$ module. Power supply of carrier wave is provided by the 5$12 \mathrm{~V}$ switch power supply, or 5V supply port of $\mathrm{CH} 340$ module.

\section{Conclusions}

The overall scheme of power testing system based on power line carrier communication is designed in this paper, and it focus on the hardware designing. In experiment, the system could achieve accurate detection data of electrical equipment by monitor computer in real time. It could use extensively in various aspects and prospects.

In overall, the power line carrier communication technology and its application is in a booming period. With the continuous development of communication technology, the power line carrier communication is bound to have a very broad application prospects.

\section{Acknowledgement}

This research is supported by Science and technology project of Xi'an city, China (CXY1524 (2)).

\section{References}

1. ZHANG Tai; GU Xiao-feng; ZHANG Dawei; WU Bin. Design of intelligent power management system based on power line carrier communication. Modern Electronics Technique, 2016(04)

2. ZHANG Yong. Hardware Design And Implementation of Power Line Carrier Communication for Medium Voltage Based on DSP. North China Electric Power University, 2015

3. Qiao Chen-xi, Chen Ke, Yang Fan, Hu Xiao-guang. Power line broadband carrier meter reading system research and collector design based on OFDM technology. (ICIEA2011),2011:2768-2773

4. HAN Yao-long. Design of Low-voltage Power Line Carrier Communication Module Based on OFDM. Harbin University of Science and Technology, 2014

5. M. Korki and N. Hosseinzaden, A channel model for power line communication in the smart grid. IEEE/PES. Power Systems Conference and Exposition. 2011, pp. 1-7

6. CHEN Feng, ZHENG Wen-gang, SHEN Chang-jun, ZHOU Ping, WU Wen-biao. Low-voltage power line carrier communication technology and its application. Power System Protection and Control, 2009.11 